\title{
Modeling thought and feelings: the why, what and whereabouts of animals in psychiatry
}

Disorders of mood and thought arise from altered functioning of highest human faculties. The idea that meaningful animal models of these disorders can be made seems utterly absurd. Are not we at the top of millennia of evolution, have not we reached levels of complexities that are out of range of any of the animal species populating this planet? Thus, we were caught by surprise when first scientists claimed that drug and gene manipulations apparently elicited disorders of mood and thought remarkably similar to ours, in rats and mice! How it can be true and what it means? And, especially, is it of any use?

To begin answering these questions, let me back off. Disorders of thought and emotion like obsessive-compulsive disorder (OCD), schizophrenia, autism, depression, arise from a complex intertwining of genetic factors and our history, including the impact of adverse environmental events. Although there is overwhelming evidence that both genes and environment play crucial roles in disease pathogenesis, how gene/environment interactions occur in any of these disorders is much more difficult to understand. To help reduce the complexity, single-gene mutations have been induced in "simple" organisms such as nematodes, fish and (more relevant for us) mice. This approach has paid off, regardless of whether it was random or targeted to potentially interesting genes. This is because in these organisms it is relatively easy to control for environmental variables, and also because experiments can be done under conditions in which virtually all the other genes in that particular animal strain are invariant, except for the one that is mutated.

The null mutation of a single gene, the so-called "gene knockout", is a remarkably powerful approach. It is a step forward with respect to traditional techniques of biological psychiatry, such as inflicting brain lesions or injecting drugs into the brain. This is because lesions inevitably destroy many cells and pathways. Virtually all pharmacological agents are not perfect agonists or antagonists for a certain receptors, and it is very difficult to manipulate intracellular molecules using drugs. Furthermore, the single gene approach promises to shed light on the ancient question of the specific role played by genes independently of the environment.

Hundreds of genes have been knocked out in the mouse genome. Gene knockouts have yielded insights into neurotransmitter receptor subtype contributions to mood and cognition. For example, several investigators reported that mice lacking the serotonin $1 \mathrm{~A}\left(5-\mathrm{HT}_{1 \mathrm{~A}}\right)$ receptor are more anxious, suggesting that this serotonin receptor subtype is responsible for the known efficacy of serotonin re-uptake inhibitors in mood disorders. ${ }^{1}$ Increased levels of dopamine caused by the knockout of the dopamine transporter result deficiencies in the gating of sensory information, manifested as pre-pulse inhibition, which is due to excessive stimulation of D2 dopamine receptors. ${ }^{2}$ Abnormal modulation of sensory input is thought to be present in schizophrenia, and these mice data explain in part the beneficial effects of D2 dopamine antagonists in psychotic patients.

Equally interesting findings have emerged in many other studies which targeted genes important for patterning of the vertebrate embryo. Mice lacking a gene called dishevelled have defects in social interactions and defects in sensory gating, which are among the characteristics present in autism. ${ }^{3}$ Dishevelled transduces, inside the cell, the signal provided by growth factors of the $W n t$ family during embryonic development; interestingly, the $W n t 2$ gene is located within the $7 \mathrm{q}$ region of the human genome that was linked to autism in all systematic studies. These analyses suggest that Wnt/dishevelled signaling may be responsible for certain features of autistic children. Other autistic features may be explained by different genes. For example, mice lacking the neuropeptide oxytocin fail to develop social memory, as defined by a decrease in olfactory investigation in repeated encounters with a conspecific mouse. ${ }^{4}$

The extrapolation of findings in rodents to humans should be done in the proper evolutionary context. Although it is truly amazing that the great majority of our genes are also present in mice, the functional effects of a given gene may be substantially different in different species. Is there a general lesson to be learned from genetic mouse models? Bad genes generally predispose to abnormal cognitive functions (anxiety, social memory, etc.) not to the acquisition of entire syndromes such as autism, schizophrenia or depression. This is in line with the notion that complex human disorders arise as a result of interactions of many different genes. Another cautionary note arise from the fact that if a gene is knocked out from the earliest stages of development, adaptive and compensatory phenomena and myriad of secondary effects occurring during development may mask its true function. Investigators are now modifying genes in given subsets of cells - technique called conditional knockout. These refined genetic interventions allow the 
probing of gene function in specific areas of the CNS and either during development or in the adult organism. This level of analysis is essential to discriminate their function during normal development and in disease states. From these new studies is emerging a surprising vulnerability of the young brain for the development of mental aberrations. For example, when the $5-\mathrm{HT}_{1 \mathrm{~A}}$ was knocked out only in adult mice, there was no development of abnormal anxiety; conversely, mice developed anxiety when this receptor was absent beginning in the early postnatal period. ${ }^{5}$ Thus, the normal role of $\mathrm{HT}_{1 \mathrm{~A}}$ receptor during development may be different from its function when this receptor is activated by therapeutic intervention in adulthood. Similar findings are emerging from our own work in mice lacking the Fibroblast Growth Factor Receptor 1, which develop stereotypic behavior as adults as a consequence of a transient disruption of this gene function during development.

We are coming to a humbling answer to our beginning question. Undoubtedly, the generation of animals with abnormal cognitive functions due to altered genes will be useful for psychiatry. First, animal models will tremendously advance our under-

\section{References}

1. Ramboz S, Oosting R, Amara DA, Kung HF, Blier P, Mendelsohn M et al Serotonin receptor 1A knockout: an animal model of anxiety-related disorder [comment]. Proc Natl Acad Sci U S A 1998;95:14476-81.

2. Ralph RJ, Paulus MP, Fumagalli F, Caron MG, Geyer MA. Prepulse inhibition deficits and perseverative motor patterns in dopamine transporter knock-out mice: differential effects of D1 and D2 receptor antagonists. J Neurosci 2001;21:305-13. standing of the interactions among different genes, and between genes and environment. More importantly, understanding the roles of these genes in brain development may allow us, one day, to reach a precise understanding of how normal and aberrant cognitive functions may develop over time, and what are the predisposing and protective factors. A powerful tool that will help the analysis of these animal models is the gene array technology. Assessing profiles of altered gene expression in these knockouts will delineate secondary molecular consequences stemming from the original gene alteration. This in turn will allow developing therapeutic strategies or can be used to predict outcome. The best possible future use these animal models will be understanding the disease process in mechanistic terms in order to develop rational treatment strategies.

Flora M Vaccarino

Child Study Center \& Section of Neurobiology, Yale University Medical School
3. Lijam N, Paylor R, McDonald MP, Crawley JN, Deng CX, Herrup K et al. Social interaction and sensorimotor gating abnormalities in mice lacking Dvl1. Cell 1997;90:895-905.

4. Ferguson JN, Young LJ, Hearn EF, Matzuk MM, Insel TR, Winslow JT. Social amnesia in mice lacking the oxytocin gene. Nat Genet 2000;25:284-8.

5. Gross C, Zhuang X, Stark K, Ramboz S, Oosting R, Kirby L et al. Serotonin $1 \mathrm{~A}$ receptor acts during development to establish normal anxietylike behaviour in the adult [comment]. Nature 2002;416:396-400. 